\title{
Computational Time Analysis of Signal Processing Algorithm-An Analysis
}

\author{
Gokul P*, Karthikeyan T* , KrishnaKumar R.* Malini S.** \\ * Final Year ECE students, Department of Electronics and Communication Engineering, \\ **Assistant Professor, Department of Electronics and Communication Engineering, SVS College Of \\ Engineering, Coimbatore
}

\begin{abstract}
Current manufacturing systems, including dedicated transfer lines and flexible manufacturing systems, do not provide adequate and most economical solutions for products with variable market demands. One of the crucial problems in manufacturing is the machine tool failure due to loss of surface material in cutting operations like drilling and milling. Proceeding with a damaged tool may result in unfit fabricated work piece. On the other hand, it is unnecessary to change the cutting tool if it is still able to continue cutting operation. Hence, an effective diagnosis mechanism is necessary for the automation of machining processes so that production loss and downtime can be avoided. This study concerns with the development of a tool wear condition-monitoring technique. Tool Wearing Monitoring technique can be established based on fuzzy logic. Fuzzy logic scheme explains Tool wearing Monitoring based on Signal processing by obtaining statistical parameters derived from thrust force, machine sound and vibration signals were used as inputs to fuzzy process and their output values serves as the input parameters to the second stage. The second stage with a remarkable threshold function, then assess the condition of the tool. Index Terms- FFT, Singular Spectrum Analysis, Tool Wearing Monitoring.
\end{abstract}

\section{Introduction}

Most natural phenomena occur continuously in time, such as the variations of temperature of the human body, forces exerted by muscles, or electrical potentials generated on the surface of the scalp. These are analogue signals, being able to take on any value (though usually limited to a finite range). They are also continuous in time, i.e. at all instants in time is their value available. However, analogue, continuous-time signals are not suitable for processing on the now ubiquitous computer-type processors (or other digital machines), which are built to deal with sequential computations involving numbers. These require digital signals, which are formed by sampling the original analogue data.

The theory of sampling was developed in the early twentieth century by Nyquist and others, and revolutionized signal processing and analysis especially from the 1960s onwards, when the appropriate computer technology became widely available. The rapid development of high-speed digital integrated circuit technology in the last three decades has made digital signal processing the technique of choice for many most applications, including multimedia, speech analysis and synthesis, mobile radio, sonar, radar, seismology and biomedical engineering. Digital signal processing presents many advantages over analog approaches: digital machines are flexible, reliable, easily reproduced and relatively cheap. As a consequence, many signal processing tasks originally performed in the analog domain are now routinely implemented in the digital domain, and others can only feasibly be implemented in digital form. In most cases, a digital signal processing systems is implemented using software on a general-purpose digital computer or digital signal processor (DSP).

Signal processing applications includes medical applications, where signal analysis has been widely applied in patient monitoring, diagnosis and prognosis, as well as physiological investigation and in some therapeutic settings (e.g. muscle and sensory stimulation, hearing aids). Early work on tool wear monitoring focused on time series methods and frequency domain analysis. With these methods, a threshold value needs to be set between the normal and abnormal tool states. However, the threshold value varies with cutting conditions. To improve the performance of tool failure sensing, more advanced methods, such as pattern recognition analysis and statistical methods have been developed. These methods have gained various degrees of success in practical applications for monitoring cutting processes. More recently, artificial neural networks (ANN) and their combination with fuzzy logic models have been extensively applied to the area of tool wear estimation. ANN has the advantages of superior learning, noise suppression and parallel computation abilities. However, successful application of an ANN-based monitoring system is strongly dependent on the proper selection of the type of network structure and needs adequate training data, which is not always available, especially for the abnormal tool state. The traditional signal processing, statistical and pattern recognition approaches generally assume that the sensor signals are stationary. However, due to the nature of manufacturing processes, the sensor signals are usually non stationary. The non-stationary nature of signals is due to nonlinearity and/or time 
dependency of the process. Thus, the approaches that deal with non-stationary signals are more appropriate for process monitoring. Many signals, including most from a mechanical based machine like lathe origin, can be classified as random: repeated recordings result in signals that are all different from each other, but share the same statistical characteristics. The power spectrum reflects some of the most interesting of these characteristics. The interpretation and estimation of the power spectral density will be addressed in the final part of this chapter.The signal processing approach in this paper is carried out with a basic Fast Fourier Transform on different tools and in identifying the best and worst tool based on sound processing. Further this approach is to be carried out with Singular Spectrum Analysis and the suitable algorithm is analyzed. This paper is organized as follows. The principle of the Fourier transform is briefly described in section 2. Following the experimental setup in section 3, Analysis of the data using the Fourier transform and SSA method are discussed in section 4. Section 7 presents some results. The conclusions are given in section 8 .

\subsection{Fast Fourier Transform:}

\section{Signal Processing Algorithms}

A fast Fourier transform (FFT) is an efficient algorithm to compute the discrete Fourier transform (DFT) and its inverse. An FFT computes the DFT and produces exactly the same result as evaluating the DFT definition directly; the only difference is that an FFT is much faster. A discrete Fourier transform can be computed using an FFT by means of the Danielson-Lanczos lemma if the number of points $N_{\text {is a power of }}$ two. If the number of points $N$ is not a power of two, a transform can be performed on sets of points corresponding to the prime factors of $N$ which is slightly degraded in speed. An efficient real Fourier transform algorithm or a fast Hartley transform (Brace well 1999) gives a further increase in speed by approximately a factor of two. Base- 4 and base- 8 fast Fourier transforms use optimized code, and can be 20-30\% faster than base-2 fast Fourier transforms. prime factorization is slow when the factors are large, but discrete Fourier transforms can be made fast for $\mathrm{N}=2,3,4,5,7,8,11,13$, and 16 using the Winograd transform algorithm.

Fast Fourier transform algorithms generally fall into two classes: decimation in time, and decimation in frequency. The Cooley-Tukey FFT algorithm first rearranges the input elements in bit-reversed order, then builds the output transform (decimation in time). The basic idea is to break up a transform of length $N$ into two transforms of length $\mathrm{N} / 2$.

The most commonly used FFT is the Cooley-Tukey algorithm. This is a divide and conquer algorithm that recursively breaks down a DFT of any composite size $\mathrm{N}=\mathrm{N}_{1} \mathrm{~N}_{2}$ into many smaller DFTs of sizes $\mathrm{N}_{1}$ and $\mathrm{N}_{2}$, along with $\mathrm{O}(\mathrm{N})$ multiplications by complex roots of unity traditionally called twiddle factors. The most well-known use of the Cooley-Tukey algorithm is to divide the transform into two pieces of size N/2 at each step, and is therefore limited to power-of-two sizes, but any factorization can be used in general (as was known to both Gauss and Cooley/Tukey). These are called the radix-2 and mixed-radix cases, respectively (and other variants such as the split-radix FFT have their own names as well). Although the basic idea is recursive, most traditional implementations rearrange the algorithm to avoid explicit recursion. Also, because the CooleyTukey algorithm breaks the DFT into smaller DFTs, it can be combined arbitrarily with any other algorithm for the DFT.

The Fast Fourier Transform (FFT) is an algorithm* for transforming data from the time domain to the frequency domain. Since this is exactly what we want a spectrum analyzer to do, it would seem easy to implement a Dynamic Signal Analyzer based on the FFT. However, we will see that there are many factors which complicate this seemingly straightforward task. First, because of the many calculations involved in transforming domains, the transform must be implemented on a digital computer if the results are to be sufficiently accurate. Fortunately, with the advent of microprocessors, it is easy and inexpensive to incorporate all the needed computing power in a small instrument package. Note, however, that we cannot now transform to the frequency domain in a continuous manner, but instead must sample and digitize the time domain input.

\subsubsection{Resolution and Range}

- FFT takes N sample points and returns N/2 frquency lines.

- Some higher-frequency lines are lost because of the anti-aliasing filter

- Highest frequency controlled by the sampling rate.

- Lowest frequency controlled by block size.

- Highest frequency $F_{a}=F_{s} / 2$ (Nyquist theorem)

- Lowest frequency $=1 / \mathrm{T}$ (block fundamental frequency)

- $\quad$ Lines evenly spaced in between. Spacing $=1 / \mathrm{T}$. 


\subsection{SOUND SIGNAL IN A CNC LATHE:}

\section{Experimental Model}

Cutting tests were performed on a $30 \mathrm{hp} \mathrm{CNC}$ lathe. An accelerometer was mounted on the cutting tool holder attached to the turret, as shown in Figure 1, to measure vibration in the feed direction. This is because preliminary results have shown the vibration signal in the feed direction to

be more sensitive than those in the cutting and radial directions in detecting tool wear. The vibration signals were first amplified using a charge amplifier and low-pass filtered with cut-off frequency of $6 \mathrm{kHz}$, and then sampled at $100 \mathrm{kHz}$ using a 12-bit data acquisition card. Every data set was $0.1 \mathrm{~s}$ in length, equivalent to $50 \mathrm{k}$ data points. Cutting started with a sharp insert and was stopped after every minute for tool wear measurement using a toolmaker's microscope. When the cutting edge develops an average flank wear height of at least $0.3 \mathrm{~mm}$ or the maximum wear height of $0.6 \mathrm{~mm}$, it is considered to be a worn out edge. This limit was chosen in accordance with the criteria recommended by ISO 3685 to define effective tool life for carbide tools [ISO,1993].

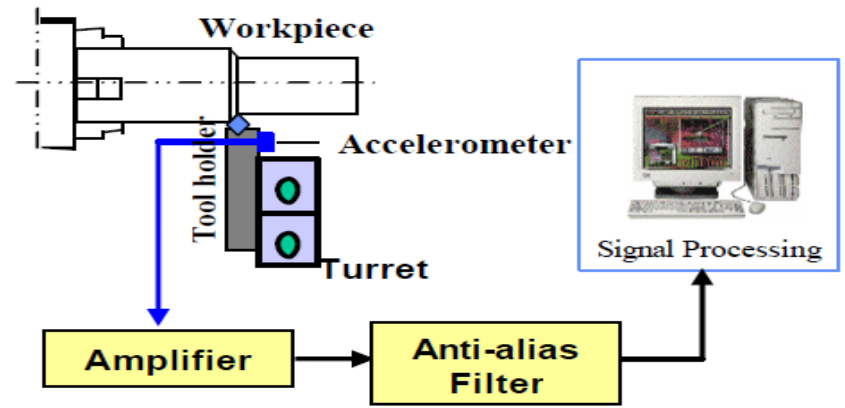

Figure 1.1 :Experimental Setup for measuring Sound Signal from a CNC Lathe

The experiments were conducted to detect two tool states, namely a sharp tool and a worn tool. The work piece material and cutting conditions are shown below

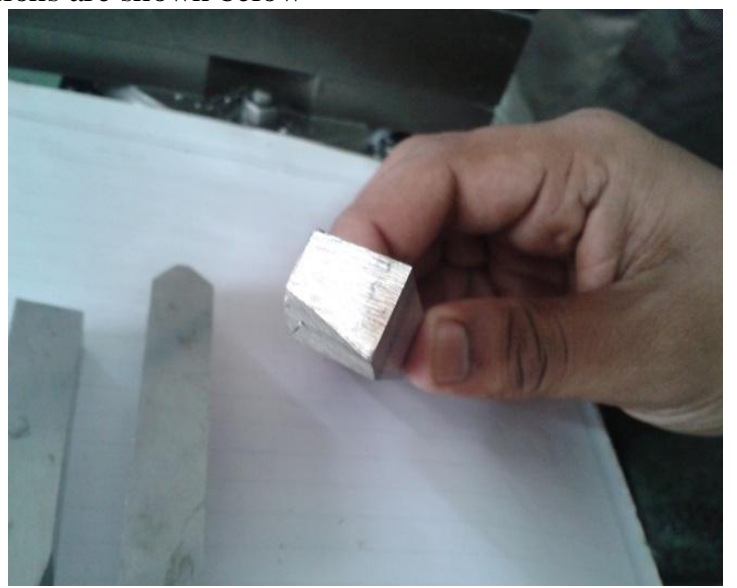

Fig (a) Good tool

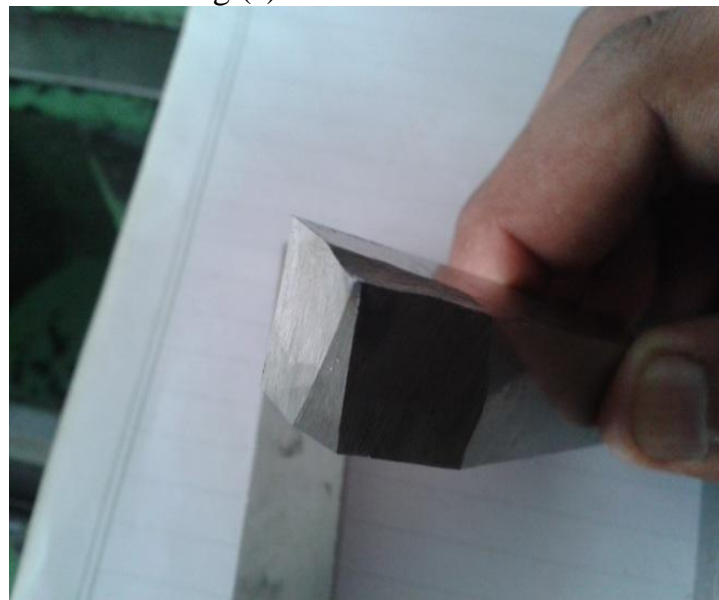

Fig (b) $20 \%$ Worn tool 


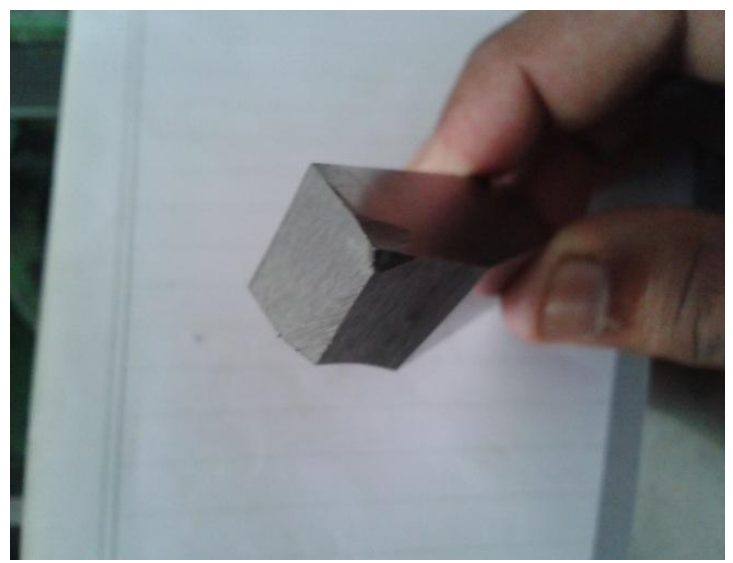

Fig (c) $40 \%$ Worn tool

IV. Methodology:

This chapter explains about the principles and process involving in the analysis. The sound is extracted by the microphone and it is recorded as a wave sound file. The amplitude of the signal determines the condition of the cutting tool. Hence it is necessary to convert into frequency domain and for this fast Fourier transform is taken to obtain the signal in frequency domain.

From the report of FFT of the given input signal is varied in amplitude with other. The sound signal is recorded at the spindle speed of 350 RPM and feed rate is $1 \mathrm{~mm}$.

The Flowchart is given below

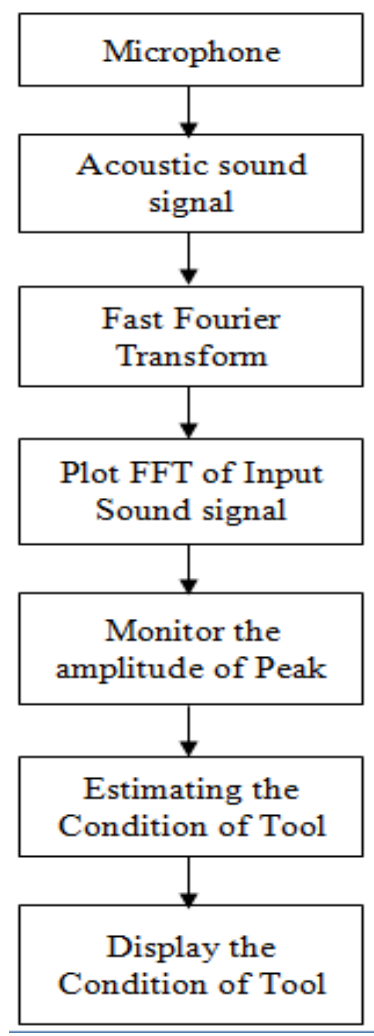

\section{Data Analysis:}

This section explains the extraction of distinct features of sensor signal using Fourier transform analysis. The effectiveness of the approach focussed are shown by the simulated results.

\subsection{Fast Fourier Transform Analysis:}

The first stage is the extraction of usefulfeatures from the bulk original data signals. Thisprocess is performed by computing the discretewavelet coefficients of the framed data signal. Todistinguish between a worn tool and a sharp tool, itis necessary to visually examine the time frequencyplanes generated by fast fourier 
transformfrom different tool states. In Figure 2, shows the tool selected for our experimental analysis. variations ofthe coefficients of the wavelet transformations are presented for a normal and a worn tool. The horizontal axis represents time. The vertical axis represents the amplitude of coefficients. Scales 1-6are equivalent to frequency bandwidths from low to high. It can be seen from Figure 2 (a) that the turning vibration signal from the sharp tool can be mainly characterized by lower frequency coefficients (lower scales 1-3). The higher scalecoefficients are very small, and can be neglected. Thus the characteristic of sharp tool signals can be represented by a small number of coefficients. This makes the wavelet transforms very efficient.The same observation can be made for a worn tool from Figure 2 (b).Referring to the same figures, it can be seen that the distribution of intensities is different for a sharp and a worn tool. The highest energy for a sharp tool concentrates on the lowest scale. However, this shifts to the next higher frequency region (higher scale) when the tool is worn out. The results presented in this paper are typical experimental results obtained so far. The work is underway to verify consistency, repeatability and reliability of the approach, and the results will be reported in the future.

\section{Tool wear estimation}

The relationship between the AE signal and tool wear is not simple. Kim et al. [18] observed the purely progressive Tools wear in turning operations. As a result, they found that in most experimental results the refined mean level (RML) of the averaged AE signal increases at first with an increase of flank wear, and then stays at an approximately constant level even with further increase of flank wear while the fluctuation of the RML across the constant level becomes rather high. Clearly, the relationship between the AE signal and tool wear condition is nonlinear, so the general mathematical relation cannot be used to map this relation. If we can look for an effective mathematical model to map the relationship between the AE signal (some features) and tool wear, the AE signal could be used to monitor tool wear condition in real time for turning.

Some models have been presented, such as a linear regression model developed to relate the flank wear of a carbide turning insert with the cumulative count of $\mathrm{AE}$, and the limiting value of the cumulative $\mathrm{AE}$ count for the limiting flank wear was predicted using this model. The AE count rate has been found to be a reliable parameter for predicting the flank wear of a cutting tool in real time. A linear regression model has been developed to relate the flank wear of a carbide turning insert with the cumulative count of AE. The correlation between intrinsic frequencies and AE sources is identified by examining the RMS, dominant amplitude, type, and count rate of the AE signals. The tool life estimated from the RMS of the AE signal is shown to be in good agreement with that determined from measurements of the maximum wears and width on the tool nose. The results obtained demonstrate that $\mathrm{AE}$ is an effective technique for in-process wear monitoring and wear mechanism identification of multilayer ceramic-coated tools. To effectively monitor different tool wear conditions, while avoiding the effect of other factors such as cutting parameters, some methodologies have been presented, as follows.

\subsection{Fuzzy classifier}

Fuzzy $c$-means algorithm is one of the most popular methods in the fuzzy classifier. In this approach, the aim in clustering is to determine the cluster centers, which are representative values of features corresponding to the classified categories. Once clustering centers are determined at the learning stage, then the classification is made by the comparison of the incoming pattern and each clustering center. Let $X=\{X 1, X 2, \ldots, X n\}_{-} R$, where each $X i=\left(x i 1, x i 2, \ldots, x i s_{-} R\right.$ is a feature vector; $x i j$ is the $j$ th feature of individual $x i$. For each integer $c, 2 \_c \_n$, let $V c n$ be the vector space of $c \cdot n$ matrices with entries in $[0,1]$, and let $u i j$ denote the $i j$ th element of any $U_{-} V c n$. The function $u i: X \rightarrow[0,1]$ becomes a membership function and is called a fuzzy subset in $X$. Here $u i j=u i(x j)$ is called the grade of membership of $x j$ in the fuzzy set $u i$. In the space of samples, we suppose that there are $n$ samples, which can be divided into $c$ classes. Consider the following subset of $V c n$ :

$\mathrm{M}_{\mathrm{fc}}=\left\{\mathrm{U} € \mathrm{~V}_{\mathrm{cn}} \mid \mathrm{u}_{\mathrm{ij}} €[0,1] \square \mathrm{i}, \mathrm{j} ; \sum \mathrm{u}_{\mathrm{ij}}=1 \square \mathrm{j} ; \sum \mathrm{u}_{\mathrm{ij}}>0 \square \mathrm{i}\right\}$

Each $U \_M f c$ is called a fuzzy $c$-partition of $X ; M \mathrm{fc}$ is the fuzzy $c$-partition space associated with $X$. For any real number $m \_[1,5]$, define the real-valued functional $J: M f \mathrm{f} \cdot L \mathrm{c} \rightarrow R$ by

$$
J(U, V)=\sum_{k=1}^{n} \sum_{i=1}^{c}\left(u_{i k}\right)^{m}\left\|x_{k}-v_{i}\right\|^{2}
$$


$1 \_m \_$, and usually $m=2$. where $U=\{u i k\}$ is the membership function, with $u i k \_[0,1]$, which denotes the degree of embership of the $k$ th pattern and $i$ th cluster centers; $V=\{v 1, v 2, \ldots, v c\}$ is a vector of $c$ clusters. These $v i$ are interpreted as clusters defined by their companion $U$ matrix, and play a fundamental role in our development. The functional $J$ is a weight, least squares objective function. In order to obtain the optimum fuzzy partition, this objective function must be minimized, i.e. minimize $\{J(U, V)\}$ The optimal solution to the above equation is that

$$
\begin{aligned}
u_{i k}= & \frac{1}{\sum_{j=1}^{c}\left(\frac{\left\|X_{k}-V_{i}\right\|}{\left\|X_{k}-V_{j}\right\|}\right)^{2 /(m-1)}}, \forall i, k \\
V_{i}= & \frac{\sum_{k=1}^{n}\left(u_{i k}\right)^{m} X_{k}}{\sum_{k=1}^{n}\left(u_{i k}\right)^{m}}, \forall i
\end{aligned}
$$

Suppose that under a given cutting condition, the features of training data sets determine a clustering center. Then all subsequent observations can be classified by using above equation. That is

$$
u_{k 0}=\frac{1}{\sum_{j=1}^{c}\left(\frac{\left\|X_{0}-V_{i}\right\|}{\left\|X_{0}-V_{j}\right\|}\right)^{2 /(m-1)}}, \forall i, k
$$

where $u k 0$ is the fuzzy grade of the current observation being assigned to the $k$ th wear state category and $X 0$ is the current observation. A wavelet packet transform is used to capture the features of the AE signal, which are sensitive to the changes in tool wear condition, but are insensitive to the variation in process working conditions and various noises. The extracted features are classified by using the fuzzy ISODATA algorithm. As a result, the tool wear condition can be estimated over a wide range of cutting conditions for boring.

\subsection{Neural networks}

Neural networks are organized in layers each consisting of neurons or processing elements that are interconnected. There are a number of learning methods to train neural nets but the back-propagation (back-prop) Paradigm has emerged as the most popular training Mechanism. The back-prop method works by measuring the difference between output and the observed output value. The values being calculated at the output layer are propagated to the previous layers and used for Adjusting the connection weights. Fig. 5 shows a typical Multi layered feed forward neural network.

A class of polynomial learning network (PLN) models is used to identify cutting tool conditions; these multilayered networks have a self-organizing control structure based on the mechanisms of exhibition and Inhibition. 
Inputs

(The features of AE signal,

cutting parameters, and others)

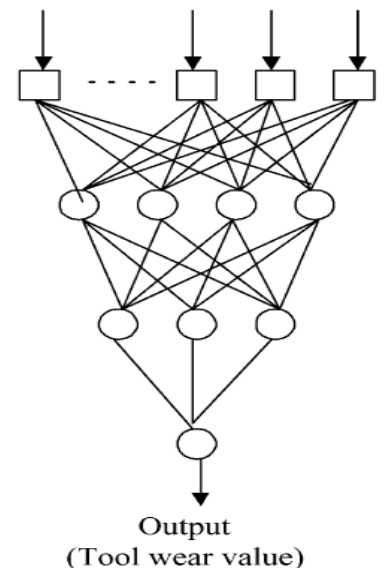

Fig. 5. Typical multilayered feedforward neural network.

AE signals from an interrupted face-turning operation are modeled, and tool evolution from normal, cracked and finally broken tools are discriminated through network connectivity and node weights. However, feed forward neural networks require expensive training information and cannot remain adaptive after training. An unsupervised ART2 neural network is used for the fusion of AE and force information and decision making of the tool flank wear state. In order to overcome neural network drawbacks, a hybrid model of neural network and fuzzy logic (fuzzy neural network) is presented. There are many possible combinations of the two systems. A fuzzy neural network $\mathrm{k}$ is used to describe the relation between the monitoring features, derived from wavelet-based AE signals, and the tool wear condition.

VII. Performance Analysis:

In this section, computer simulated results is provided to illustrate the performance of sound signal obtained through FFT over the different tools selected.

Table: SIMULATION PARAMETERS

\begin{tabular}{|l|l|}
\hline No. of Transmit Antennas & 2 \\
\hline No. of receive Antennas & 2 \\
\hline Modulation Scheme & BPSK \\
\hline Channel Model & AWGN \\
\hline Equalizer & ZF,ML,MMSE,MMSE- \\
& SIC \\
\hline
\end{tabular}

\subsection{The performance of the $20 \%$ worn tool:}

The Zero forcing equalizer is not much suitable. To say, it achieves diversity but doubles the data rate.It is clear from the following zero forcing equalization, the channel for symbol transmitted from each spatial antenna is similar to a $1 \times 1$ Rayleigh fading channel. Hence it is not much suitable equalizer though very much simpler than all other equalizers. 

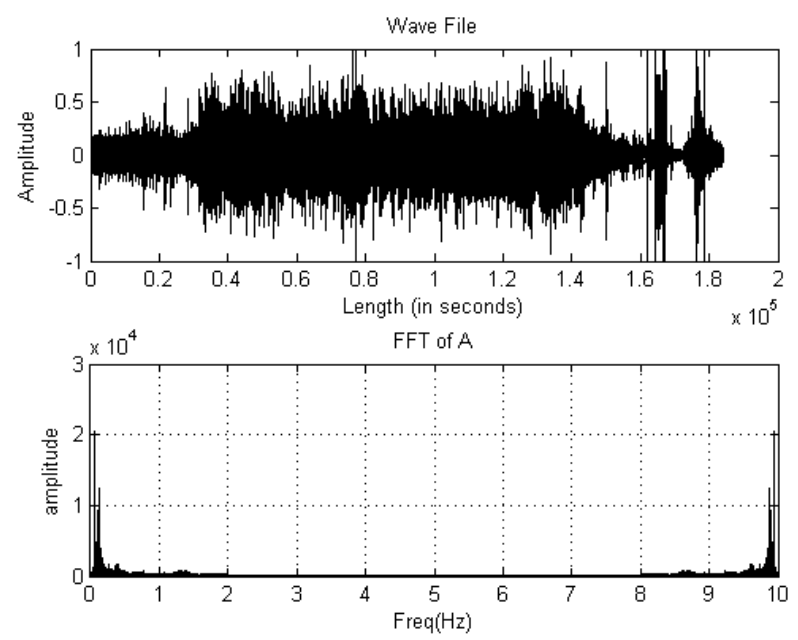

Fig.5.1: Performance of $20 \%$ worn tool

\subsection{The performance of $40 \%$ worn tool:}
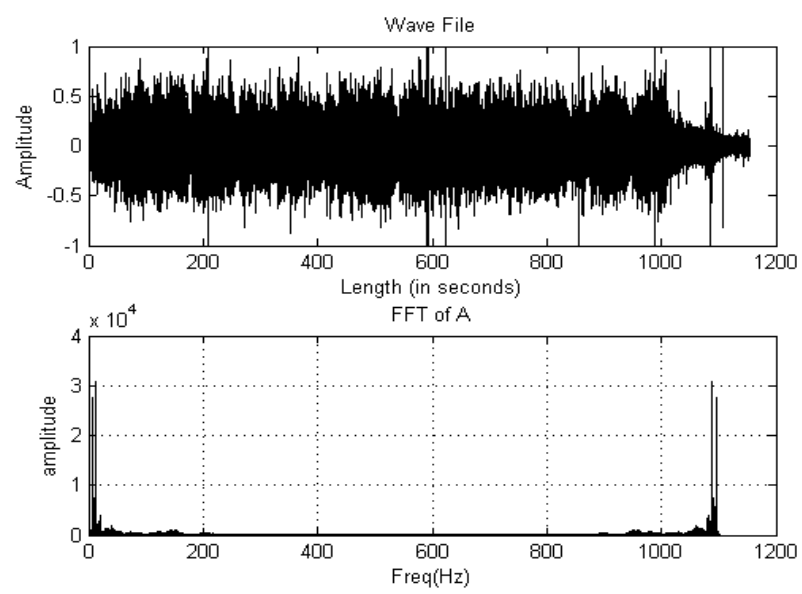

Fig.5.2: Performance of $40 \%$ worn tool

The results for $2 \times 2$ MIMO with Maximum Likelihood (ML) equalization obtained is closely matching the 1X2 antenna of Maximum Ratio Combining(MRC) type.Of all the equalizer types Maximum Likelihood offers better BER. The ML equalizer is optimal since it minimizes the probability of a sequence error. Further, this equalizer requires knowledge of the channel characteristics and statistical distribution of noise corrupting the signal to compute the metrics for decision making.

\subsection{The performance of Good tool:}

The results for $2 \times 2$ MIMO MMSE equalization shows a $3 \mathrm{~dB}$ improvement when compared to zero forcing equalizer. When noise term is zero, MMSE equalizer reduces to ZF equalizer.
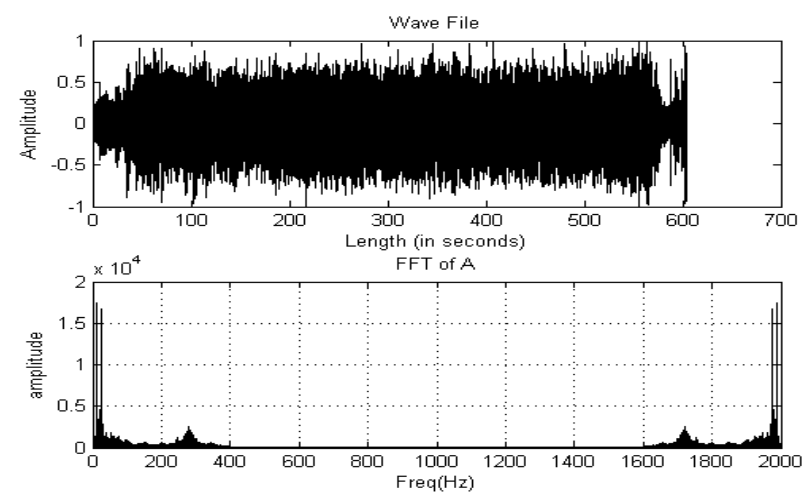

Fig.5.3: Performance of Good tool 

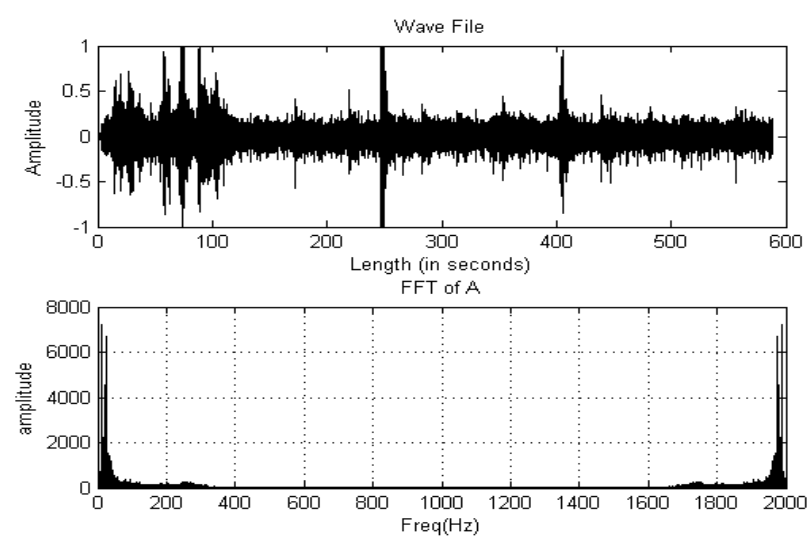

Fig.5.4: Performance without tool

The amplitude and the Condition of the tool is tabulated below

\begin{tabular}{|l|l|l|l|}
\hline Tool & $\begin{array}{l}\text { Spindle } \\
\text { Speed } \\
(\text { RPM })\end{array}$ & $\begin{array}{l}\text { Feed } \\
\text { rate } \\
(\mathrm{mm})\end{array}$ & Amplitude $(\mathrm{dB})$ \\
\hline Good tool & 350 & 1 & 17500 \\
\hline $20 \%$ Worn tool & 350 & 1 & 21000 \\
\hline $40 \%$ Worn tool & 350 & 1 & 31000 \\
\hline Without tool & 350 & 1 & 7500 \\
\hline
\end{tabular}

The above mentioned information's are the average of no of experimental values. At the specified spindle speed, there are 5 experiments are taken with same tool for analyzing.

From the tabulation clearly shows that without tool the lathe machine exhibits very low amplitude and the good tool of lathe machine exhibits slight increase in amplitude. If the tool is worn then its amplitude crosses above $20000 \mathrm{~dB}$ at initial frequency and as like $40 \%$ worn tool exhibits amplitude increased to $31000 \mathrm{~dB}$. By this we can say the condition of tool clearly.

For estimation so many techniques like Neural network or Fuzzy control logics are used.

\section{CONCLUSION:}

In this paper, a combined approach for the acoustic signal processing for identifying tool wearing condition is attempted. Here we considered the basic FFT algorithm of signal processing, whose performance is evaluated based on computational time analysis . As it is known,Signal processing is an important measure in many applications including medicine, multimedia compression, machineries. We had chosen machineries in which tool wearing is to be monitored for seemless profitable outcome of an industry. The purpose of monitoring the computational time is to prevent the damage of product as soon as possible. The result performance of FFT with two transmit and receive antennas, the MMSE equalization results with improvement in BER of around $10^{-3}$. The performance of these different equalizers for two transmit and two receive antennas has been tested with simulations. It was observed that among the different equalizers, MMSE equalizer when combined with Successive Inference Canceller was able to provide unambiguous tracking after applying the temporal filter and enhance the signal quality. The effectiveness of this paper work to provide maximum signal gain in the presence of several interference sources was shown using simulated data.

[1] BrankaVucetic\&Jinhong Yuan 'Space TimeProcessing'

[2] MohinderJankiraman, 'Space Time Codes \& MIMO Systems'

[3] John G. Proakis. "Digital Communication”, Tata McGraw Hill, New York.

[4] S.M.Alamouti, 'A simple transmit diversity technique for wireless communication' IEEE J.Selected Areas Communication, Volume 16,pp 1451-1458, Oct 1998.

[5] S. Bittner, E. Zimmermann, and G. Fettweis, "Low Complexity SoftInterference Cancellation for MIMOSystems," in IEEE VehicularTechnology Conference (VTC), 2006.

[6] J. Tellado, L.M.C. Hoo, and J.M. Cioffi, "Maximum- LikelihoodDetection of Nonlinearly Distorted Multicarrier Symbols by IterativeDecoding," IEEE Transactions on Communications, vol. 51, no. 2, pp.218-228, 2003

[7] A. Burg, M. Rupp_, N. Felber, W. Fichtner, "Practical Low Complexity Linear Equalization for MIMO-CDMA Systems" IEEE proceedings of the 37thAsilomar Conference on Signals, Systems \& Computers, Nov 2003.

[8] The MathWorksInc.,http://www.mathworks.com 
[9] UweTrautwein, Tad Matsumoto and Christian Schneider John G. Proakis. "Reiner Thoma "Exploring the Performance of Turbo MIMOEqualization in Real Field Scenarios", Fifth International Symposium on Wireless Personal Multimedia Communications (WPMC '2002), Honolulu, Hawaii on October 27-30, 2002

[10] Digital Communication", Tata McGraw Hill, New York.

[11] P. Grbec, P. Leskovar, Acoustic emission of a cutting process, Ultrasonics 15 (1) (1977) 17-20.

[12] K. Iwata, T. Moriwaki, Application of acoustic emission measurement to in-process sensing of tool wear, Ann. CIRP 26 (1-2) (1977) 19-23.

[13] K. Uehara, Identification of chip formation mechanism through acoustic emission measurement, Ann. CIRP 33 (1) (1984) $71-74$

[14] K. Kojima, I. Inasaki, R. Miyake, Monitoring of turning process with acoustic emission signals, Nippon Kikai Gakkai Ronbunshu, C Hen 52 (474) (1986) 799-805.

[15] Y. Naerheim, A. Arora, In-process monitoring of machining using acoustic emission, in: Review of Progress in Quantitative Nondestructive Evaluation, 3 (B), Plenum Press, USA, 1984, pp. 753-762.

[16] T.Moriwaki, M. Tobito, New approach to automatic Detection of life of coated tool based on acoustic emission measurement, American Society of Mechanical Engineers,Production Engineering Division (Publication) PED 33 (1988) 75-82.

[17] . Ravindra, Y. Srinivasa, R. Krishnamurthy, Acoustic emission for tool condition monitoring in metal cutting, Wear 212 (1) (1997) 78-84.

[18] S. Liang, D. Dornfeld, Tool wear detection using time series analysis of acoustic emission, J. Eng. Ind. Trans. ASME 111 (3) (1989) 199-205.

[19] E. Chung, Y. Chiou, S. Liang, Tool wear and chatterdetection in turning via time-series modeling and frequency band averaging, Manuf. Sci. Eng. Am. Soc. Mech. Eng. PED 64 (1993) 351-358.

[20] X. Li, Z. Yuan,Tool wear monitoring with wavelet packet transform-fuzzy clustering method, Wear 219 (2) (1998) 145-154.

[21] S. Cho, K. Komvopoulos, Correlation between acoustic emission and wear of multi-layer ceramic coated carbide tools, J. Manuf.Sci. Eng. Trans. ASME 119 (2) (1997) 238-246.

[22] E. Emel, E. Kannatey-Asibu, Tool failure monitoring in turning by pattern recognition analysis of AE signals, J. Manuf. Sci. Eng.Trans. ASME 110 (2) (1988) 137-145.

[23] E. Emel, E. Kannatey-Asibu, Acoustic emission and force sensor fusion for monitoring the cutting process, Int. J. Mech. Sci. 31(1112) (1989) 795-809.

[24] S. Damodarasamy, S. Raman, Inexpensive system for classifying tool wear states using pattern recognition, Wear 170 (2) (1993)149-160.

[25] S.T. Farlow, Self-organizing Methods in Modeling, Marcel Dekker, New York, 1984.

[26] C. Jiaa, D. Dornfeld, Self-organizing approach to the prediction and detection of tool wear, ISA Trans. 37 (4) (1998) $239-255$.

[27] S. Darenfed, S. Wu, Polynomial learning networks for cutting tool diagnosis in machining operations, Trans. Can. Soc. Mech.Eng. 16 (2) (1992) 147-163.

[28] Y. Niu, Y. Wong, G. Hong, Intelligent sensor system approach for reliable tool flank wear recognition, Int. J. Adv. Manuf. Technol.14 (2) (1998) 77-84.

[29] E. Emel, Tool wear detection by neural network based acoustic emission sensing, American Society of Mechanical Engineers,Dynamic Systems and Control Division (Publication) DSC 28 (1991) 79-85.

[30] Y.X. Yao, X. Li, Z.J. Yuan, Tool wear detection with fuzzy classification and wavelet fuzzy neural network, Int. J. Machine Tools \& Manufacture 39 (1999) 1525-1538.

[31] S. Rangwala, D. Dornfeld, Integration of sensors via neural networks for detection of tool wear states, American Society of Mechanical Engineers, Production Engineering Division (Publication) PED 25 (1987) 109-120.

[32] E. Chung, Y. Chiou, S. Liang, Tool wear and chatter detection in turning via time-series modeling and frequency band averaging, American Society of Mechanical Engineers, Production Engineering Division (Publication) PED 64 (1993) 351-358.

[33] X.Q. Li, Y. Wong, A.Y.C. Nee, Comprehensive identification of tool failure and chatter using a parallel multi-ART2 neural network,J. Manuf. Sci. Eng. Trans. ASME 120 (2) (1998) 433-442.

[34] G. Byrne, D. Dornfeld, I. Inasaki, G. Ketteler, W. Konig, R. Teti, Tool condition monitoring (TCM) - The status of research and industrial application, Ann. CIRP 44 (2) (1995) 541-567.

[35] X. Li, Intelligent tool condition monitoring using wavelet and fuzzy neural network, PhD thesis, Harbin Institute of Technology, Dec. 1997.

[36] A. Menon, Z. Boutaghou, Time-frequency analysis of tribologicalsystems-Part I: implementation and interpretation, Tribol. Int 31 (9) (1998) 501-510.

[37] E. Serrano, M. Fabio, Application of the wavelet transform to acoustic emission signals processing, IEEE Trans. Signal Process. 44 (5) (1996) 1270-1275.

[38] C. Heil, D. Walnut, Continuous and discrete wavelet transforms, SIAM Rev. 31 (4) (1989) 628-666.

[39] S. Kamarthi, S. Kumara, P. Cohen, Wavelet representation of acoustic emission in turning process, Intell. Eng. Syst. Artif. Neural Netw. 5 (1995) 861-866. 